Claudia Akemi Shibuya Teshima ${ }^{1}$, Mirian Watanabe ${ }^{1}$, Sandra Hideko Nakamura $^{1}$, Maria de Fátima Fernandes Vattimo ${ }^{2}$

1. Pesquisadora do Laboratório Experimental de Modelos Animais (LEMA) da Escola de Enfermagem da Universidade de São Paulo - USP - São Paulo, Brasil.

2. Livre docente, Professora da Escola de Enfermagem e Laboratório Experimental de Modelos Animais (LEMA) da Escola de Enfermagem da Universidade de São Paulo - USP - São Paulo, Brasil.

Trabalho realizado no Laboratório Experimental de Modelos Animais (LEMA) da Escola de Enfermagem da Universidade de São Paulo.

Submetido em 26 de Julho de 2010 Aceito em 8 de Setembro de 2010

Autor para correspondência: Maria de Fátima Fernandes Vattimo Av. Dr. Enéas de Carvalho Aguiar, 419 Cerqueira César CEP: 05403-000 - São Paulo (SP), Brasil.

Fone: (11) 3061-8837 / Fax: (11) 30617546

E-mail: nephron@usp.br

\section{Efeito renoprotetor da estatina: modelo animal de isquemia-reperfusáo}

\author{
Renoprotective effect of statin: a ischemia-reperfusion animal \\ model
}

\section{RESUMO}

Objetivo: A lesão renal aguda isquêmica, de causa multifatorial, apresenta morbidade e mortalidade alarmantes. A tem demonstrado papel renoprotetor, com componente antioxidante, antiinflamatório e vascular. A atividade de heme oxigenase-1 pode ser mediadora desses efeitos pleitrópicos da estatina sobre o rim, ou seja, independente da ação de redução de lipídio. Esse estudo visou avaliar se o efeito renoprotetor da estatina pode ter mecanismo heme de proteçáo em ratos.

Métodos: $\mathrm{O}$ modelo isquêmico foi obtido por meio do clampeamento dos pedículos renais bilaterais por 30 minutos, seguido de reperfusão. Foram utilizados ratos Wistar, machos, pesando entre 250$300 \mathrm{~g}$, distribuídos nos seguintes grupos: SHAM (controle, sem clampeamento renal); Isquemia; Iquemia+Estatina (sinvastatina $0,5 \mathrm{mg} / \mathrm{kg}$, via oral por $3 \mathrm{dias}$ ); Isquemia+Hemin (indutor de $\mathrm{HO}-1,1$ $\mathrm{mg} / 100 \mathrm{~g}$, intraperitoneal $24 \mathrm{~h}$ antes da ciestatina, inibidor de HMG-CoA redutase, rurgia); Isquemia+SnPP (inibidor de $\mathrm{HO}$ $1,2 \mu \mathrm{mol} / \mathrm{kg}$ intraperitoneal $24 \mathrm{~h}$ antes da cirurgia); Isquemia+Estatina+Hemin e Isquemia+Estatina+SnPP. Foram avaliados a função renal (clearance de creatinina, Jaffé), osmolalidade urinária, peróxidos urinários e a imunohistoquímica para ED-1.

Resultados: Os resultados mostraram que a estatina melhorou a função renal, a osmolalidade urinária, reduziu a excreção de peróxidos urinários e a infiltração de macrófagos em rins de animais submetidos à isquemia renal. $\mathrm{O}$ indutor da heme oxigenase-1 e a sua associação com sinvastatina reproduziram o padrão de melhora determinado pela sinvastatina.

Conclusáo: $\mathrm{O}$ estudo confirmou $\mathrm{o}$ efeito renoprotetor da estatina, com ação antioxidante e antiinflamatória, e sugere que esse efeito tenha interface com o sistema heme de proteção renal.

Descritores: Rim/lesóes; Sinvastatina; Inibidores de hidroximetilglutaril-CoA redutases; Heme oxigenase-1; Ratos Wistar

\section{INTRODUÇÁO}

A isquemia por hipotensão ou sepsis é uma das causas mais frequentes de lesão renal aguda isquêmica (LRAi) em pacientes críticos. No período pós transplante pode representar a perda do enxerto. A LRAi envolve uma cascata de eventos com alteraçôes da hemodinâmica glomerular, lesão tubular, ativação da resposta inflamatória e liberação de espécies reativas de oxigênio (EROs). ${ }^{(1)}$ Modelos in vivo de isquemia/reperfusão (I/R) renal são utilizados para elucidar mecanismos fisiopatológicos ou implementar terapias farmacológicas de proteção renal. Nesse contexto, a sinvastatina tem sido considerada por suas açóes pleitrópicas renais caracterizadas como antiinflamatória, antioxidante e de proteçáo vascular. Ressalte-se que pleitrópicas se caracterizam por ações independentes da ação lipolítica primária. Evi- 
dências recentes confirmam a prevenção de reaçóes vasculares inflamatórias em estudos clínicos e em modelos animais após a administraçáo de estatinas. ${ }^{(2)}$

Mecanismos de proteção e adaptação celular, como a enzima heme oxigenase-1 (HO-1), são induzidos frente ao insulto isquêmico. A heme oxigenase é uma enzima limitadora que degrada a heme, facilitando a sua conversão para biliverdina. Essa conversão, que necessita de fosfato dinucleotídeo adenina nicotinamida (NADPH), libera metal ferroso e produz monóxido de carbono que, pela ação da biliverdina redutase a biliverdina, é convertido em bilirrubina. Todas as moléculas resultantes desta reação são liberadas em quantidades equimolares e possuem funçóes antioxidante, antiinflamatória, anti-apoptótica e possivelmente modulatória do sistema imune. Estudos com modelos animais de nefrotoxicidade e de isquemia renal confirmaram que o efeito protetor desta enzima pode ser resultante da liberação de subprodutos. ${ }^{(3)}$

A ausência de possibilidades clínicas de resgate ou prevenção da lesão renal aguda (LRA) em pacientes graves gera desconforto e indignação diante das estatísticas desfavoráveis. Esse cenário estimula o desenvolvimento de estudos que visem elucidar os mecanismos precisos envolvidos na lesão, em que interferências farmacológicas, como podem ser a estatina e o sistema heme oxigenase, venham a representar alternativa no tratamento. De fato, a ocorrência de lesão súbita, como é o caso da LRA, pode engessar procedimentos de prevenção. Contudo, a estatina tem demonstrado resultados significativos também após dose única administrada 30 minutos antes da lesão, reforçando seu desempenho promissor mesmo em situaçóes agudas. ${ }^{(4)}$ Este estudo teve como objetivo verificar a ação protetora renal da sinvastatina no modelo animal de I/R e avaliar se a mediação pela enzima heme-oxigenase 1 pode estar presente.

\section{MÉTODOS}

Todos os procedimentos realizados neste estudo estáo de acordo com os Princípios Éticos de Experimentação Animal adotado pelo Colégio Brasileiro de Experimentação Animal (COBEA) e foram aprovados pela Comissão de Ética em Experimentação Animal do Instituto de Ciências Biológicas da Universidade de São Paulo. Todos os animais tiveram acesso livre à água e alimentos permanecendo em condiçôes térmicas com ciclos de dia e noite durante todo o experimento.

Foram utilizados ratos Wistar, machos e adultos, pesando entre 250 - 300 gramas divididos nos seguintes grupos: SHAM - simulação do ato cirúrgico, Isquemia - clampeamento bilateral dos pedículos renais durante 30 minutos, Isquemia + estatina - isquemia renal em animais pré condicionados durante 3 dias com Sivascor ${ }^{\oplus}$-Baldacci $0,5 \mathrm{mg} / \mathrm{kg}$ por gavagem (v.o.), Isquemia + Hemin - isquemia renal e pré condicionamento com
Hemin-Sigma, indutor de HO- 1 , $1 \mathrm{mg} / 100 \mathrm{~g}$, intraperitoneal (i.p.), 24 horas antes da cirurgia, Isquemia + protoporfirina de estanho (SnPP) - isquemia renal e pré condicionamento com SnPP-Sigma, inibidor seletivo de HO- $1,2 \mu \mathrm{mol} / \mathrm{kg}$, intraperitoneal (i.p.), 24 horas antes da cirurgia, Isquemia + estatina + Hemin - isquemia renal e pré condicionamento com sinvastatina e Hemin, Isquemia + estatina + SnPP - isquemia renal e pré condicionamento com sinvastatina e $\mathrm{SnPP}$.

Os animais foram anestesiados com tiopental de sódio 40 - $50 \mathrm{mg} / \mathrm{kg}$, i.p. e submetidos à laparotomia para clampeamento bilateral dos pedículos renais durante 30 minutos e restabelecimento da perfusão renal. Os animais foram colocados em gaiolas metabólicas para coleta de urina de 24 horas para estudo de função renal (FR) e estresse oxidativo. Após este período, os animais foram anestesiados novamente com tiopental de sódio $60 \mathrm{mg} / \mathrm{kg}$ para nova laparotomia e coleta de sangue por meio da punçáo da aorta abdominal para estudos de função renal. $\mathrm{O}$ rim esquerdo de cada animal foi retirado para estudos de imunohistoquímica.

\section{Funçáo renal}

Avaliada pelo clearance de creatinina por meio da mensuração de creatinina plasmática e urinária realizada pelo método de Jaffé. ${ }^{(5)}$

\section{Funçáo tubular}

Mensuração da osmolalidade urinária por meiodo osmômetro - Advanced Osmometer ${ }^{\circ}$ - model 3D3.

\section{Estresse oxidativo}

Mensuração dos níveis de peróxidos urinários (PU) por meio do método FOX-2. A mensuração dos níveis de PU é considerada biomarcador da geração de peróxidos de hidrogênio e preditor da extensão de estresse oxidativo em modelos experimentais in vivo. ${ }^{(6,7)}$

\section{Imunohistoquímica}

Lâminas de coloração imunohistoquímica com uso de anticorpo monoclonal anti ED-1 (macrófago e monócitos) - Serotec.

\section{Análise estatística}

Utilizou-se o método GLM (Anova univariada) e teste de comparações múltiplas ajustados para Bonferrone. Os valores $\mathrm{p}<0,05$ foram considerados significantes.

\section{RESULTADOS}

A tabela 1 mostra a redução significativa da filtração

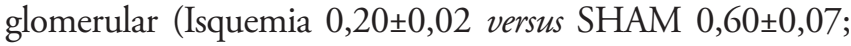


$\mathrm{p}<0,05$ ) e função tubular (Isquemia $766 \pm 188$ versus SHAM $1793 \pm 191 ; \mathrm{p}<0,05)$ nos animais submetidos à isquemia renal de 30 minutos quando comparados com o grupo controle SHAM, confirmando o modelo de LRAi, com manutençáo do fluxo urinário. $\mathrm{O}$ pré condicionamento com sinvastatina, o indutor de HO-1 e associação sinvastatina e indutor demonstraram melhora da FR com elevação dos valores de

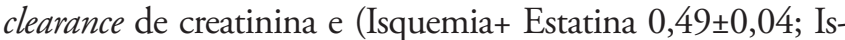
quemia + Hemin 0,46 0,03 ; Isquemia + Estatina + Hemin $0,54 \pm 0,07$ versus Isquemia $0,20 \pm 0,02 ; \mathrm{p}<0,05)$ e função tubular (Isquemia + Estatina 1153 \pm 404 ; Isquemia + Hemin $1013 \pm 211$; Isquemia + Estatina + Hemin $1314 \pm 394$ versus Isquemia $766 \pm 188 ; \mathrm{p}<0,05)$. A administração do inibidor HO-1 e a associação sinvastatina e inibidor de HO-1 induziram elevação dos valores de clearance de creatinina, porém na comparação entre os tratamentos associados prevaleceu a diferença estatística em favor ao Hemin, indutor de HO-1 (Isquemia + Estatina + Hemin 0,54 $\pm 0,07$ versus Isquemia + Estatina + SnPP 0,36 $\pm 0,07 ; \mathrm{p}<0,05)$.

A tabela 2 apresenta valores de PU. Observou-se que o grupo de animais submetidos à isquemia renal apresentou valores de PU superiores ao grupo controle, confirmando o envolvimento do mecanismo oxidativo nesse modelo de lesão (Isquemia $13,5 \pm 0,8$ versus SHAM 5,6 $\pm 0,9 ; \mathrm{p}<0,05$ ). Os animais submetidos à isquemia e pré condicionados com sinvastatina ou com o indutor de HO-1 responderam com redução dos valores de PU em relaçáo ao grupo Isquemia (Isquemia+ Estatina 7,9 $\pm 1,0$; Isquemia + Hemin $7,6 \pm 1,0$ versus Isquemia $13,5 \pm 0,8 ; p<0,05)$. Os grupos que receberam $\mathrm{SnPP}$ ou a associação farmacológica de indutor ou inibidor com sinvastatina demonstraram níveis de PU superiores ao SHAM ( $\mathrm{p}<0.05)$,

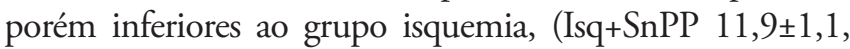

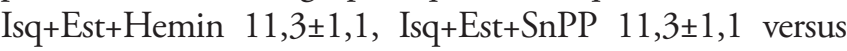
SHAM 5,6 $\pm 0,9$ versus Isq $13,5 \pm 0,8$ )

A tabela 3 mostra os valores quantitativos do infiltrado de macrófagos nos rins dos animais dos grupos em que foram empregadas intervenções farmacológicas. Observou-se redução dos valores de infiltrado nos grupos em que administrou
Tabela 2 - Valores de peróxidos urinários dos diversos grupos

\begin{tabular}{lcc}
\hline Grupos & $\mathrm{N}$ & $\begin{array}{c}\text { Peróxidos urinários } \\
\text { (nmol/g de creatinina) }\end{array}$ \\
\hline SHAM & 8 & $5,6 \pm 0,9$ \\
Isquemia & 8 & $13,5 \pm 0,8^{\mathrm{a}}$ \\
Isquemia+Estatina & 8 & $7,9 \pm 1,0^{\mathrm{b}}$ \\
Isquemia+Hemin & 5 & $7,6 \pm 1,0^{\mathrm{b}}$ \\
Isquemia+SnPP & 5 & $11,9 \pm 1,1^{\mathrm{a}}$ \\
Isquemia+Estatina+Hemin & 6 & $11,3 \pm 1,1^{\mathrm{a}}$ \\
Isquemia+Estatina+SnPP & 8 & $11,3 \pm 1,1^{\mathrm{a}}$ \\
\hline
\end{tabular}

${ }^{a} \mathrm{p}<0,05$ versus SHAM; ${ }^{b} \mathrm{p}<0,05$ versus Isquemia. Dados representados em média \pm desvio padráo.

Tabela 3 - Quantificaçáo do infiltrado de macrófagos por imunohistoquímica dos diversos grupos

\begin{tabular}{lc}
\hline Grupos & Macrófagos por campo \\
$\mathrm{N}=4$ & $2,90 \pm 0,60$ \\
\hline Isquemia & $0,50 \pm 0,06$ \\
Isquemia+Estatina & $0,60 \pm 0,05$ \\
Isquemia+Hemin & $0,80 \pm 0,05$ \\
Isq+SnPP & $1,60 \pm 0,05$ \\
Isquemia+Estatina+Hemin & $2,80 \pm 0,05$ \\
Isquemia+Estatina+SnPP &
\end{tabular}

Dados representados em média \pm desvio padrão.

sinvastatina, hemin, SnPP e estatina + hemin, porém , sem significância estatística.

\section{DISCUSSÁO}

Apesar de inúmeros avanços na pesquisa e na clínica, a LRA isquêmica continua sendo um dos principais indicadores de mau prognóstico funcional, tanto em rins nativos quanto em rins transplantados. Sabe-se que o mecanismo isquêmico de lesão renal determina a deposição do complexo antígeno-anticorpo no glomérulo e induz ativação e infiltração de neutrófilos que determinam a resposta inflamatória. As células mesangiais respondem a esse mecanismo irritativo

Tabela 1 - Funçáo renal global dos diversos grupos

\begin{tabular}{lcccc}
\hline Grupos & $\mathrm{N}$ & $\begin{array}{c}\mathrm{FU} \\
(\mathrm{ml} / \mathrm{min})\end{array}$ & $\begin{array}{c}\text { Clcr 100g } \\
(\mathrm{ml} / \mathrm{min})\end{array}$ & $\begin{array}{c}\text { Osmolalidade urinária } \\
(\mathrm{mOsm})\end{array}$ \\
\hline SHAM & 6 & $0,006 \pm 0,001$ & $0,60 \pm 0,07$ & $1793 \pm 191$ \\
Isquemia & 7 & $0,013 \pm 0,008$ & $0,20 \pm 0,02^{\mathrm{a}}$ & $766 \pm 188^{\mathrm{a}}$ \\
Isquemia+Estatina & 10 & $0,016 \pm 0,011$ & $0,49 \pm 0,04^{\mathrm{b}}$ & $1153 \pm 404^{\mathrm{a}}$ \\
Isquemia+Hemin & 8 & $0,013 \pm 0,003$ & $0,46 \pm 0,03^{\mathrm{b}}$ & $1013 \pm 211^{\mathrm{a}}$ \\
Isquemia+SnPP & 9 & $0,010 \pm 0,005$ & $0,33 \pm 0,04^{\mathrm{a}}$ & $977 \pm 125^{\mathrm{a}}$ \\
Isquemia+Estatina+Hemin & 6 & $0,008 \pm 0,004$ & $0,54 \pm 0,07^{\mathrm{b}}$ & $1314 \pm 394^{\mathrm{b}}$ \\
Isquemia+ Estatina+SnPP & 8 & $0,012 \pm 0,009$ & $0,36 \pm 0,07^{\mathrm{abc}}$ & $1218 \pm 274^{\mathrm{b}}$ \\
\hline
\end{tabular}

FU - fluxo urinário. ${ }^{a} \mathrm{p}<0,05$ versus SHAM; ${ }^{b} \mathrm{p}<0,05$ versus Isquemia; ${ }^{c} \mathrm{p}<0,05$ versus Isquemia+Estatina+Hemin. Dados representados em média \pm desvio padrão. 
secretando eicosanóides, proteases e EROs. ${ }^{(8)}$ Vários estudos demonstram efeito benéfico de protetores vasculares, vasodilatadores e antioxidantes, destacando-se as estatinas pelos efeitos pleirotrópicos antiinflamatório e antioxidante. ${ }^{(9)}$

Esse estudo confirmou o efeito benéfico de proteção renal do pré condicionamento com sinvastatina na LRAi em modelo animal, constatado por meio do aumento na filtração glomerular, redução da lesão tubular e redução dos níveis de PU de animais que foram subsequentemente submetidos à isquemia renal de 30 minutos. Estudos em modelos de I/R em animais demonstram o efeito renoprotetor da sinvastatina com melhora da necrose tubular aguda verificada por meio de análise histológica e redução dos valores de fração de excreção de sódio. ${ }^{(2)} \mathrm{O}$ uso de pravastatina em modelos de I/R em ratos também confirmou redução da resposta inflamatória pela mensuração dos níveis de IL-6. ${ }^{(10)}$ Na prática clínica, estudos evidenciaram menor incidência de LRA após realização de cirurgias cardíacas com uso de estatinas no período pré operatório e nos dois dias consecutivos a cirurgia. ${ }^{(11)}$

Diferentes mecanismos de proteção celular são induzidos durante a I/R renal, incluindo a heat shock protein 32, conhecida também como isoforma induzível de HO-1. A enzima HO-1 exerce importante papel na manutenção da homeostase renal. ${ }^{(12)} \mathrm{O}$ pré condicionamento com Hemin, indutor de HO-1, confirmou proteção renal antioxidante nesse modelo de I/R caracterizado por melhora da FR e reduçáo dos níveis de PU. O efeito benéfico do indutor de HO-1 parece estar principalmente associado à formação de subprodutos como a bilirrubina, ferritina e monóxido de carbono. A geração de bilirrubina em quantidades equimolares confere proteção antioxidante, bem como a formação de ferritina, que inativa o ferro na cascata de lesão oxidativa. O monóxido de carbono atua com um potente vasodilatador. ${ }^{(13)}$

A associação dos tratamentos sinvastatina e indutor de HO-1 não apresentou resultados superiores quando comparada com o tratamento isolado. A estratégia de associação farmacológica visou verificar se os resultados vistos na função renal, tubular e peroxidação seriam superiores, inferiores ou permaneceriam similares aos tratamentos isolados. A ausência de diferenças entre isolados e associados faz crer que haja uma provável similaridade catalítica entre a sinvastatina e HO-1 e que, uma vez saturados pelos efetores, os receptores em comum não desencadeiam resposta adicional ou diversa. Estudos realizados anteriormente confirmaram que a estatina está envolvida com a indução de $\mathrm{HO}-1$ em vários órgãos aos níveis de trancrição, translação e catalização, o que ressalta os resultados de interação vistos no estudo ora apresentado. ${ }^{(14)}$

Há que se considerar ainda que a super expressão da eNOS e dos níveis de NO tem sido relacionados a renoproteção desempenhada pela sinvastatina. Sendo de enfática relevância, vale ressaltar que evidências demonstram que os efeitos protetores da $\mathrm{HO}-1$ ou dos produtos de degradação do heme intensificam a funçáo endotelial por meio da interferência na biodisponibilidade do NO. Foram descritos três mecanismos celulares que ilustram esse movimento: $\mathrm{HO}$ e os produtos heme modulam a expressáo e a atividade do $\mathrm{NO}$, previnem a inativação do $\mathrm{NO}$ vascular e compensam a perda do $\mathrm{NO}$ vascular. Dessa forma, esse estudo reforça que o efeito HO-1 como mediadora da açáo renoprotetora da sinvastatina sobre a função renal na lesão $\mathrm{I} / \mathrm{R}$ deve ser considerado e infere que a açáo do NO pode estar envolvida nesse contexto. Outros estudos avaliando a atividade dessas enzimas, associadas, trarão mais dados a respeito dessa interface celular.

Os resultados dos estudos com imunohistoquímica sugeriram que a ação antiinflamatória de ambos, estatina e HO-1, esteve presente no modelo de I/R . Contudo, o pequeno infiltrado inflamatório encontrado, mesmo no grupo isquemia, pressupóe que o período de isquemia possa não ter sido suficientemente agressivo para a obtenção de achados mais exuberantes que pudessem sublinhar o dano histológico e destacar o efeito antiinflamatório desses agentes no estudo.

Sumariamente, os resultados aqui apresentados reforçaram o efeito protetor da sinvastatina em modelo de I/R renal de 30 minutos com melhora da FR, redução dos mediadores de lesáo oxidativa e da resposta inflamatória. Esse efeito pleiotrópico da sinvastatina demonstrou ter possível interface com a enzima heme oxigenase 1 , supondo que esse seja um modelo heme de renoproteção. Estudos sobre a ação do NO e sua interferência no sistema $\mathrm{HO}$ na presença da sinvastatina serão providentes para a elucidação desses vias ainda incompreendidas.

\section{ABSTRACT}

Objective: Ischemic acute kidney injury (iLRA), with multifatorial cause, presents alarming morbidity and mortality. Statin, HMG-CoA inhibition reductase has shown a renoprotective effect, with antioxidant, antiinflamatory and vascular actions. The heme oxygenase-1 (HO-1) can be involved in these pleitropic effects of statin on the renal function. This study was performed in order to evaluate if the renoprotective effect of the statin is a heme mechanism of protection in rats.

Methods: The ischemic model was reproduced by through clamping the bilateral renal pedicles for 30 minutes followed by reperfusion. Adult Wistar rats, weighting from 250-300g, were divided into the following groups: SHAM (control); Ischemia (30 minutes renal ischemia); Ischemia+Statin (sinvastatin $0.5 \mathrm{mg} / \mathrm{kg}$,orally (v.o.) for 3 days); Ischemia+Hemin (Hemin, $1.0 \mathrm{mg} / 100 \mathrm{~g}$, intraperitoneal (i.p.), 24 hours before surgery); Ischemia+SnPP (SnPP 
$2 \mu \mathrm{mol} / \mathrm{kg}$, i.p., 24 hours before surgery ); Ischemia+Statin+Hemin; Ischemia+Statin+SnPP. RF (clearance of creatinine, Jaffé method), urinary peroxides (UP), urinary osmolality (UO) and immunohistochemical for ED-1 were evaluated.

Results: Results showed that sinvastatin ameliorated RF, urinary osmolality, reduced the UP excrection and the macrophage infiltration in rats submitted to renal ischemia. The inducer of HO-1 and its association with sinvastatin induced a similar pattern of improvement of renal function.

Conclusion: the study confirmed the renoprotective effect of the statins on renal function, with antioxidant and antiinflamatory actions, and it suggests that this effect can have an interface with the heme system of renal protection.

Keywords: Kidney/injuries; Simvastatin; HydroxymethylglutarylCoA reductase inhibitors; Heme oxygenase-1; Rats, Wistar

\section{REFERÊNCIAS}

1. Ikeda M, Prachasilchai W, Burney-Taney MJ, Rabb H, Yokota-Ikeda N. Ischemic acute tubular necrosis models and drug discovery; a focus on cellular inflammation. Drug Discov Today. 2006;11(7-8):364-70.

2. Todorovic Z, Nesic Z, Stojanovic R, Basta-Jovanovic G, Radojevic-Skodric S, Velickovic R, et al. Acute protective effects of simvastatin in the rat model of renal ischemiareperfusion injury: it is never too late for the pretreatment. J Pharmacol Sci. 2008;107(4):465-70.

3. Hill-Kapturczak N, Chang SH, Agarwal A. Heme oxygenase and the kidney. DNA Cell Biol. 2002;21(4):307-21.

4. Nesic Z, Todorivic Z, Stojanoviic R, Basta-Jovanovic G, Radojevic-Skodric S, Velickovic R, et al. Single-dose intravenous simvastatin treatment attenuates renal injury in an experimental model of ischemia-reperfusion in the rat. J Pharmacol Sci. 2006;102(4):413-17.

5. Dórea EL, Yu L, De Castro I, Campos SB, Ori M, Vaccari EM, et al. Nephrotoxicity of amphotericin B is attenuated by solubilizing with lipid emulsion. J Am Soc Nephrol. 1997;8(9):1415-22.

6. Long LH, Evans PJ, Halliwell B. Hydrogen peroxide in human urine: implications for antioxidant defense and redox regulation. Biochem Biophys Res Commun. 1999;262(3):605-9.

7. Wolff SP. Ferrous ion oxidation in presence of ferric ion indicator xylenol orange for measurement of hydroperoxides. Methods Enzymol. 1994;233:182-9.
8. Heyman SN, Rosenberger C, Rosen S. Experimental ischemia-reperfusion: biases and myths - the proximal vs. distal hypoxic tubular injury debate revisited. Kidney Int. 2010;77(1): 9-16.

9. Chatterjee PK. Novel pharmacological approaches to the treatment of renal ischemia-reperfusion injury: a comprehensive review. Naunyn Schmiedebergs Arch Pharmacol. 2007;376(1-2):1-43.

10. Sharyo S, Yokota Ikeda N, Mori M, Kumagai K, Uchida $\mathrm{K}$, Ito $\mathrm{K}$, et al. Pravastatin improves renal ischemiareperfusion injury by inhibiting the mevalonate pathway. Kidney Int. 2008;74(5):577-84.

11. Billings FT 4th, Pretorius M, Siew ED, Yu C, Brow NJ. Early postoperative statin therapy is associated with a lower incidence of acute kidney injury after cardiac surgery. J Cardiothorac Vasc Anesth. 2010. [Epub ahead of print].

12. Sikorski EM, Hock T, Hill-Kapturczak N, Agarwal A. The story so far: Molecular regulation of the heme oxygenase-1 gene in renal injury. Am J Physiol Renal Physiol. 2004;286(3):F425-41.

13. Miyazano M, Garat C, Morris KG Jr, Carter EP. Decreased renal heme oxygenase-1 expression contributes to decreased renal function during cirrhosis. Am J Physiol Renal Physiol. 2002;283(5):F1123-31.

14. Hsu M, Muchova L, Morioka I, Wong RJ, Schröder H, Stevenson DK. Tissue-specific effects of statins on the expression of heme oxygenase- 1 in vivo. Biochem Biophys Res Commun. 2006;343(3):738-44. 